\title{
Synthesis of and structure-property relationships in zinc complexes of bis-metaphenylene semiquinone biradical species
}

\author{
Joseph C. Sloop ${ }^{a *}$, David A. Shultz ${ }^{b *}$, Tashni Coote ${ }^{b}$, Benjamin Shepler ${ }^{a}$, \\ Ubie Sullivan $^{\mathrm{b}}$, Jeff W. Kampf ${ }^{\mathrm{c}}$ and Paul D. Boyle
}

\begin{abstract}
The design of novel, functionalized bis-metaphenylene semiquinone (SQ) ligands and their corresponding metal complexes which combine conformational flexibility and electron-withdrawing, electron-donating, and conjugating substituents enable investigation of multiple structure-property relationships. Along these lines, we report the synthesis of three new bis( $\mathrm{Zn}^{\mathrm{I}}(\mathrm{SQ}) \mathrm{Tp}^{\mathrm{Cum}, \mathrm{Me})}$ complexes containing the bis-metaphenylene coupling fragment. Using electron paramagnetic resonance spectroscopy, $a b$ initio computations and superconducting quantum interference device magnetometry, we show how spin-density is affected by the bis-metaphenylene system substituents. Copyright $\odot 2011$ John Wiley \& Sons, Ltd.

Supporting information may be found in the online version of this paper.
\end{abstract}

Keywords: bis(metaphenylene) semiquinone biradicals; electron paramagnetic resonance; SQUID magnetometry; substituent effects

\section{INTRODUCTION}

New paramagnetic ligands are of general interest in both organic and inorganic chemistry as components of sensors, switches, and molecular spintronics. ${ }^{[1-8]}$ Previous research in our group has shown that both conformation and substituents can modulate spin density and exchange coupling in $\operatorname{Bis}\left(\mathrm{Zn}^{\text {II }}(\mathrm{SQ}) \mathrm{Tp}^{\mathrm{Cum}, \mathrm{Me}}\right)$ biradical ligand complexes. ${ }^{[9-11]}$ The design of novel, functionalized semiquinone (SQ) ligands containing electron-withdrawing, electron-donating, and conjugating substituents enables investigation of multiple structure-property relationships and building blocks for new materials. In an effort to analyze the nature of the substituent effects observed in the bis( $\mathrm{Zn}^{\text {II }}(\mathrm{SQ}) \mathrm{Tp}^{\mathrm{Cum}, \mathrm{Me}}$ ) complexes, a broader range of substituents was required to round out the series initially studied by us, where $\mathrm{X}=t-\mathrm{Bu}, \mathrm{N}$ $\left(\mathrm{CH}_{3}\right)_{2}$, and $\mathrm{NO}_{2}{ }^{[1]}$ Therefore, the unsubstituted case $(\mathrm{X}=\mathrm{H})$ was prepared as a reference, a conjugating substituent $(X=P h)$ was selected, as well as the $\pi$-electron donor/ $\sigma$-acceptor substituent $\left(\mathrm{X}=\mathrm{OCH}_{3}\right)$ (Fig. 1).

\section{RESULTS AND DISCUSSION}

\section{Synthesis}

To acquire these new ligand species and zinc complexes, the synthetic strategy shown in Scheme 1 was employed. Yields at each step are provided. The bis( $\left.\mathrm{Zn}^{\text {II }}(\mathrm{SQ}) \mathrm{Tp}^{\mathrm{Cum}, \mathrm{Me}}\right)$ complexes were prepared using proven synthetic methodology: ${ }^{[1]}$ (1) Suzuki coupling to form compounds $\mathbf{3 a - c}$, (2) deprotection to the catechols $\mathbf{4 a - c}$ and (3) complexation to form compounds $\mathbf{5 a - c}$. Full details of the experimental procedures are found in the supplemental material.

Characterization of the Bis(Zn"(SQ)Tp $\left.{ }^{\text {Cum,Me }}\right)$ complexes The bis(Zn"(SQ)Tp ${ }^{\text {Cum,Me}}$ ) complexes $\mathbf{5 a - c}$ were characterized by several means. Absorptions in the infrared (IR) spectra readily identify the Tp $\mathrm{Tum}^{\mathrm{Cu}, \mathrm{Me}}$ ligand $\left(\sim 2540 \mathrm{~cm}^{-1}, v_{\mathrm{B}-\mathrm{H}}\right)$ and the SQ $\left(\sim 1450 \mathrm{~cm}^{-1}, v_{\mathrm{C}=0}\right)$ functionality. Also, observation of an $n \rightarrow \pi^{*}$ transition from $10,000-15,000 \mathrm{~cm}^{-1}$ in the UV-Vis spectra verifies the SQ moiety. ${ }^{[1]}$ The triplet biradical complexes were readily identified by electron paramagnetic resonance (EPR), and X-ray

\footnotetext{
* Correspondence to: J. Sloop, School of Science and Technology, Georgia Gwinnett College 1000 University Center Lane, Lawrenceville, GA 30043, USA. E-mail: jsloop@ggc.edu

* Address questions concerning the NSF funding for this work to Dr. David A. Shultz at Department of Chemistry, North Carolina State University, PO Box 8204, Raleigh, NC 27695, USA.

a J. C. Sloop, B. Shepler

School of Science and Technology, Georgia Gwinnett College, 1000 University Center Lane, Lawrenceville, GA, 30043, USA

b D. A. Shultz, T. Coote, U. Sullivan, P. D. Boyle Department of Chemistry, North Carolina State University, Raleigh, NC, 27695, USA

C J. W. Kampf

Department of Chemistry, University of Michigan, Ann Arbor, MI 48109, USA
} 
<smiles>[X]c1cc(-c2cc3c(c(C(C)(C)C)c2)O[Z17]([H])O3)cc(-c2cc3c(c(C(C)(C)C)c2)O[Z17]([H])O3)c1</smiles>

Figure 1. Substituted bis(Zn"(SQ)Tp $\left.{ }^{\text {Cum,Me }}\right)$ complexes

crystallographic data confirm the structures of the complexes. Finally, superconducting quantum interference device (SQUID) magnetometry measured the magnetic susceptibility of the complexes, from which values for $\boldsymbol{J}$ were calculated.

\section{Spectroscopy}

Infrared and UV-Vis absorptions of the bis(Zn"(SQ)Tp ${ }^{\text {Cum,Me }}$ ) complexes prepared in Scheme 1 were found to be consistent with those reported earlier. ${ }^{[1]}$ Critical absorption energies are shown below in Table 1.

Electron paramagnetic resonance spectroscopic measurements of the bis( $\left.\mathrm{Zn}^{\text {"I}}(\mathrm{SQ}) \mathrm{Tp}^{\mathrm{Cum}, \mathrm{Me}}\right)$ complexes as both frozen matrices $(77 \mathrm{~K})$ and as solutions at room temperature ( $298 \mathrm{~K})$ yield two important but different pieces of information. Because molecular tumbling is minimized in frozen matrices, electron-electron (i.e., dipole-dipole) interactions dominate the EPR spectra and are manifested as the $\Delta m_{\mathrm{s}}=1$ transition. ${ }^{[4]}$ In contrast, the random tumbling of molecules in a fluid zeros out dipolar interactions, allowing hyperfine coupling interactions to dominate the EPR spectra at room temperature and gives rise to the $\Delta m_{\mathrm{s}}=2$ transition.

Shown in Fig. 2 are the results of EPR experiments. Immediately above each experimental spectrum is the simulated spectrum obtained via the WINEPR SIMFoNIA fitting program. ${ }^{[3]}$ The experimental spectra and zero-field splitting (ZFS) parameters recorded in Table 1 are in agreement with the simulations and are also consistent with spectra obtained from bis(Zn"(SQ) $\mathrm{Tp}^{\mathrm{Cum}, \mathrm{Me}}$ ) complexes reported earlier. ${ }^{[1,12]}$ These findings, when taken together, suggest successful formation of triplet bis(semiquinone) biradical species. In the case of $\mathbf{5 b}$, it was unclear as to why the transition was broadened relative to those of other species.

\section{Magnetometry}

Superconducting quantum interference device magnetometry measurements were carried out to ascertain both the maximum spin (saturation) values $\left(M_{\mathrm{sat}}\right)$ and the dependence of the complexes' molar magnetic susceptibility $\left(\chi_{M}\right)$ on temperature as a function of substituent. For these biradicals, we observed $M_{\text {sat }}$ to approach a maximum value of 2.0 Bohr magnetons (Fig. 3), corresponding to two unpaired electrons, as the magnetic field strength was increased at $\sim 4 \mathrm{~K}$. Likewise, $\chi_{\mathrm{M}} \cdot T$ values were observed to approach their maximum value of $1.0 \mathrm{emu} \cdot \mathrm{K} / \mathrm{mol}$ at low temperature, where population of the higher energy singlet state does not occur to a large extent. As the temperature was raised during the experiment, increasing population of the higher lying singlet state reduced the triplet contribution to $\chi_{M}$, causing $\chi_{M} \cdot T$ values to approach $0.75 \mathrm{emu} \cdot \mathrm{K} / \mathrm{mol}$. These findings are consistent with earlier SQUID magnetometry data

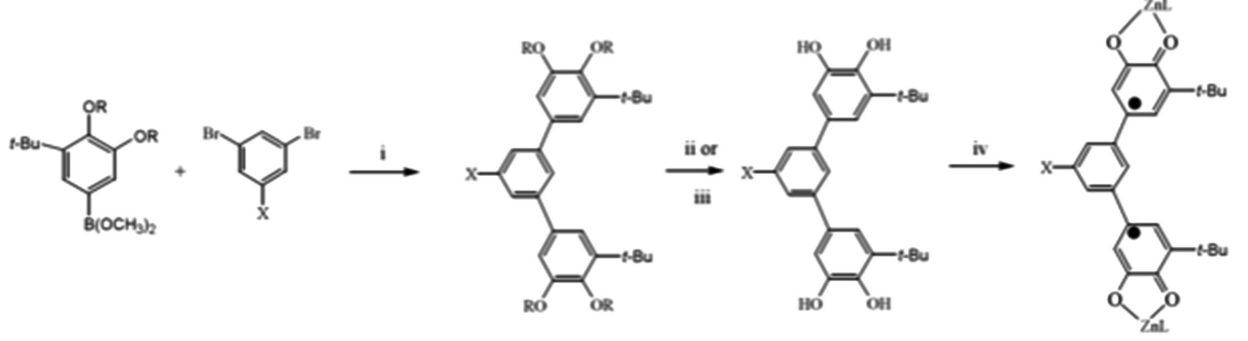

\begin{tabular}{llccc} 
& & 3a-c & 4a-c & 5a-c \\
\hline $1 \mathbf{a}\left(\mathrm{R}=\mathrm{CH}_{3}\right)$ & $2 \mathbf{a}(\mathrm{X}=\mathrm{H})$ & $\%$ Yield & \% Yield & \% Yield \\
$1 \mathbf{a}\left(\mathrm{R}=\mathrm{CH}_{3}\right)$ & $2 \mathbf{b}(\mathrm{X}=\mathrm{Ph})$ & 73 & 91 & 83 \\
$1 \mathbf{b}\left(\mathrm{CH}_{2} \mathrm{OCH}_{3}\right)$ & $2 \mathbf{c}\left(\mathrm{X}=\mathrm{OCH}_{3}\right)$ & 60 & 93 & 83 \\
\hline
\end{tabular}

Conditions: i: Boronic Ester (2eq), phenyl halide (leq), Pd( $\left.\mathrm{PPh}_{3}\right)_{4}(5-14 \mathrm{~mol} \%), \mathrm{C}_{2} \mathrm{H}_{9} \mathrm{OH}(6-15 \mathrm{~mL}), 2 \mathrm{M} \mathrm{Na} \mathrm{CO}_{3}(9-18 \mathrm{~mL}), \mathrm{THF}$ (50mL), reflux (20-30h), ii: $\mathrm{BBr}_{3}(3-4 \mathrm{eq}), \mathrm{CH}_{2} \mathrm{Cl}_{2}(20 \mathrm{~mL}),-78^{\circ} \mathrm{C} \rightarrow \mathrm{RT}, 18 \mathrm{~h}$. iii: $\mathrm{CH}_{3} \mathrm{OH}(45 \mathrm{~mL}), \mathrm{HCl}(0.1 \mathrm{~mL})$, reflux (24h), iv: (1) $\mathrm{Zn}\left(\mathrm{ClO}_{4}\right)_{2} 6 \mathrm{H}_{2} \mathrm{O}$ (2eq), $\mathrm{KTp}{ }^{\mathrm{Cm}} \mathrm{Ne}$ (2eq), $\mathrm{CH}_{3} \mathrm{OH}(4.5 \mathrm{~mL}), \mathrm{CH}_{2} \mathrm{Cl}_{2}(30 \mathrm{~mL}),(0.5-1 \mathrm{~h}) ;(2) \mathrm{N}\left(\mathrm{C}_{2} \mathrm{H}_{5}\right)_{3}$ (1.5h); (3) open to air, $18 \mathrm{~h}, \mathrm{RT}$. Notes: "Satisfactory conversion required addition of another aliquot of $\mathrm{Pd}$ catalyst and base after $24 \mathrm{~h}$. " $\mathrm{L}=\mathrm{Tp}$ " cimite

Scheme 1. Bis(Zn"(SQ)Tp $\left.{ }^{\text {Cum,Me }}\right)$ complex synthetic sequence

Table 1. Spectroscopic frequencies for bis(Zn"(SQ)Tp ${ }^{\mathrm{Cum}, \mathrm{Me})}$ complexes

\begin{tabular}{|c|c|c|c|c|c|c|}
\hline \multirow[b]{2}{*}{ Compound } & \multicolumn{2}{|c|}{ IR frequencies } & \multicolumn{2}{|c|}{ UV-Vis transitions } & \multicolumn{2}{|c|}{ EPR ZFS parameters } \\
\hline & $\mathrm{B}-\mathrm{H}\left(\mathrm{cm}^{-1}\right)$ & $\mathrm{SQ}\left(\mathrm{cm}^{-1}\right)$ & $\mathrm{n} \rightarrow \pi^{*}\left(\mathrm{~cm}^{-1}\right)$ & $\Delta \mathrm{v}_{\mathrm{n}} \rightarrow \pi *\left(\mathrm{~cm}^{-1}\right)$ & $D / h c\left(\mathrm{~cm}^{-1}\right)$ & $E / h c\left(\mathrm{~cm}^{-1}\right)$ \\
\hline $5 a$ & 2536 & 1440 & 13,086 & 0 & 0.00399 & 0.000017 \\
\hline $5 b$ & 2547 & 1440 & 13,080 & -6 & 0.00372 & 0.000042 \\
\hline $5 c$ & 2547 & 1439 & 14,060 & 974 & 0.00398 & 0.000076 \\
\hline
\end{tabular}


collected on analogous compounds. ${ }^{[1]}$ Strength of exchange coupling can be determined by fitting the magnetic data to a field-independent van Vleck expression (using $\mathrm{H}=-2 J \hat{S}_{1} \cdot \hat{\mathrm{S}}_{2}$ ), Eqn $1,{ }^{[4,5]}$

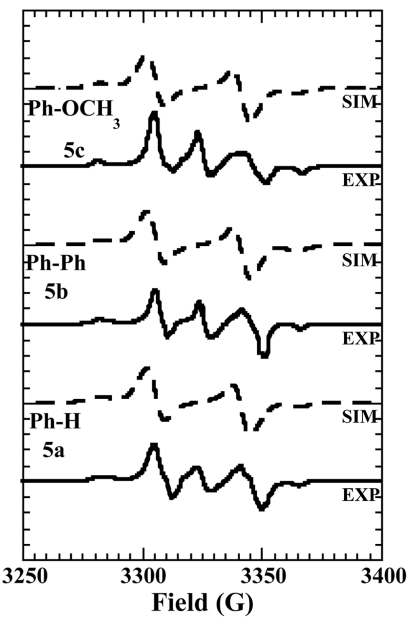

a. $\Delta \mathrm{m}_{\mathrm{s}}=1$ transition $(77 \mathrm{~K})$.

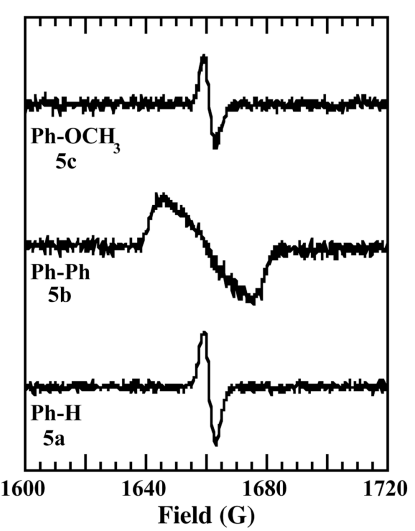

b. $\Delta \mathrm{m}_{\mathrm{s}}=\mathbf{2}$ transition $(298 \mathrm{~K})$.
Figure 2. $\operatorname{Bis}\left(\mathrm{Zn}^{\text {II }}(\mathrm{SQ}) \mathrm{Tp} \mathrm{Cum}^{\mathrm{Cu}, \mathrm{Me}}\right) \mathrm{EPR} \Delta m_{\mathrm{s}}=1$ and $\Delta m_{\mathrm{s}}=2$ transitions

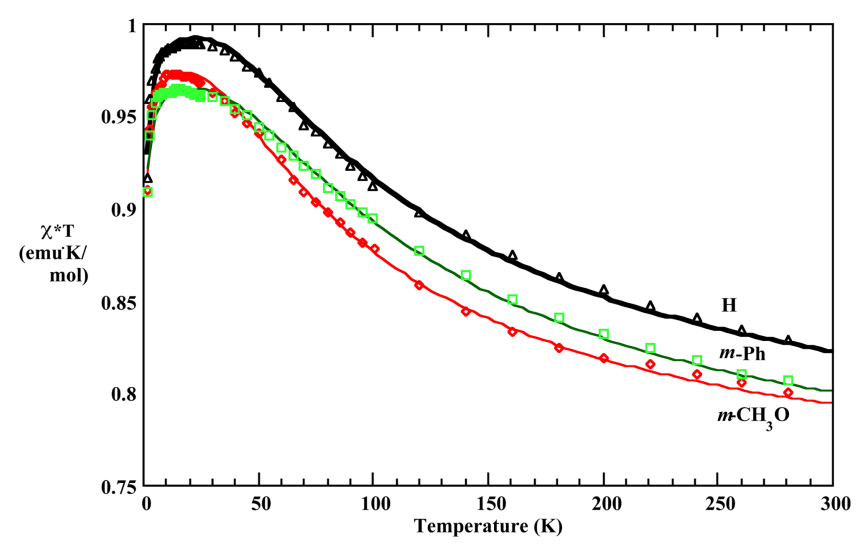

Figure 3. $\operatorname{Bis}\left(\mathrm{Zn}^{\prime \prime}(\mathrm{SQ}) T \mathrm{p}^{\mathrm{Cum}, \mathrm{Me}}\right)$ complex $\chi T$ versus $T$

$$
\chi T=\frac{2 N g^{2} \beta^{2}}{k\left[3+e^{-2 J / k T}\right]}
$$

where $g$ is the isotropic Landé constant $(g=2.0023), \beta$ is the Bohr magneton, $T$ is the temperature in Kelvin, $k$ is Boltzmann's constant, $\boldsymbol{J}$ is the intramolecular exchange coupling parameter $\left(2 J=\Delta E_{\mathrm{ST}}\right)$, and $\hat{\mathrm{S}}_{1}$ and $\hat{\mathrm{S}}_{2}$ are the spin operators for the semiquinones. The decrease of the $\chi T$ data at very low temperatures (below 5K) was accounted for with a Weiss correction, using the expression $\chi_{\text {eff }}=\chi /(1-\vartheta \chi)$, where $\vartheta=2 z J^{\prime} /\left(N g^{2} \beta^{2}\right) .{ }^{[6]}$ The origin of $J$ ' can be ZFS, intermolecular interactions, saturation effects, or some combination of these. ${ }^{[7]}$ The curve fit results are seen in Table 2. For comparison, values for complexes prepared by Shultz et al. are also included.

\section{X-ray crystallography}

The structures of complexes $\mathbf{5} \mathbf{a}-\mathbf{c}$ were verified by X-ray crystallography. Crystal structures are shown in Fig. 4 and the crystallographic parameters are provided in Table 3. Complete crystallographic files may be found in the supporting information and the crystallographic data files have been deposited with the Cambridge Crystallographic Data Center.

\section{Substituent effects study}

Spectroscopic, magnetometric, and crystallographic properties of these complexes may permit elucidation of substituent effects. These new compounds allow expansion of our investigation of substituent effects on exchange coupling between SQ moieties, ${ }^{[9]}$ to include substituted-SQ-paramagnetic metal ion exchange coupled species.

Hancock and Clague showed that shifts in the $n \rightarrow \pi^{*}$ transitions in the electronic absorption spectra of monoradical species are linearly related to $\sigma$ values. ${ }^{[13]}$ Variation in the ZFS parameters $D$ and $E$ measured by EPR can also provide information on changes in spin density distribution. $A b$ initio computations can yield additional insights on the changes in SQ spin density distribution brought about by substituents. Changes in $\boldsymbol{J}$ values obtained through SQUID magnetometry as a function of the substituent may also correlate to Hammett parameters. Finally, examination of the SQ ring torsion angles obtained through X-ray

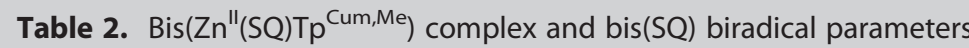

\begin{tabular}{|c|c|c|c|c|c|c|c|}
\hline \multirow[b]{2}{*}{ Compound (Substituent) } & \multicolumn{2}{|c|}{ Torsion angles } & \multicolumn{2}{|c|}{ Exchange parameters } & & \multicolumn{2}{|c|}{$\operatorname{Bis}(S Q)^{b}$ spin density } \\
\hline & $\varphi_{1}\left({ }^{\circ}\right)$ & $\varphi_{2}\left({ }^{\circ}\right)$ & $J\left(\mathrm{~cm}^{-1}\right)$ & $z J^{\prime}\left(\mathrm{cm}^{-1}\right)$ & $\mathrm{C}_{7}, \mathrm{C}_{8}{ }^{\mathrm{C}}$ & $\Sigma\left[C_{1-6}\right]^{d}$ & $\Sigma[\text { substituent }]^{d}$ \\
\hline $5 \mathbf{a}^{\mathrm{b}}(\mathrm{H})$ & 38.3 & 27.2 & 46.0 & -0.25 & $0.072,0.089$ & 0.197 & NA \\
\hline $5 \mathbf{b}^{\mathrm{b}}(\mathrm{Ph})$ & 37.6 & 41.9 & 47.4 & -0.005 & $0.096,0.093$ & 0.222 & 0.0257 \\
\hline $5 c^{\mathrm{b}}\left(\mathrm{CH}_{3} \mathrm{O}\right)$ & 33.3 & 30.8 & 35.9 & -0.07 & $0.104,0.092$ & 0.222 & 0.0016 \\
\hline $5 \mathbf{d}^{\mathrm{a}}\left(\mathrm{NO}_{2}\right)$ & 33.3 & 33.5 & 31.0 & -0.07 & $0.085,0.091$ & 0.221 & 0.0096 \\
\hline $5 e^{a}\left(\mathrm{~N}\left(\mathrm{CH}_{3}\right)_{2}\right)$ & 36.0 & 31.0 & 34.9 & -0.11 & $0.089,0.098$ & 0.214 & 0.0040 \\
\hline $5 \mathbf{f}^{\mathrm{a}}(t-\mathrm{Bu})$ & 30.2 & 30.8 & 59.3 & -0.11 & $0.080,0.095$ & 0.204 & 0.0019 \\
\hline $\begin{array}{l}{ }^{a} \text { See Ref. }{ }^{[1]} \text {. } \\
{ }^{b} \text { This work. } \\
{ }^{c} \text { a spin density. } \\
{ }^{d} a+\beta \text { spin density. }\end{array}$ & & & & & & & \\
\hline
\end{tabular}




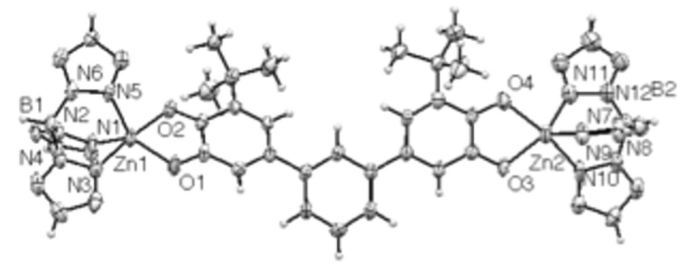

5a
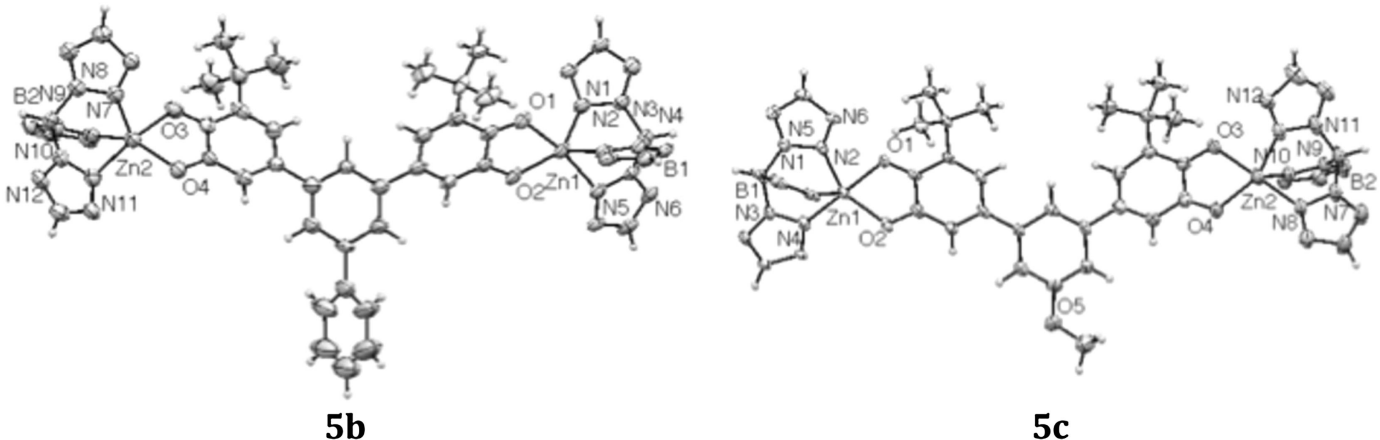

$5 c$

Figure 4. $\operatorname{Bis}\left(\mathrm{Zn}(\mathrm{SQ}) T p^{\mathrm{Cum}, \mathrm{Me}}\right)$ complex $\chi T$ ORTEP drawings (ellipsoids are at the $50 \%$ probability level and selected atoms were omitted for clarity)

\begin{tabular}{|c|c|c|c|}
\hline Compound & $5 a$ & $5 b$ & $5 c$ \\
\hline Empirical formula & $\mathrm{C}_{104} \mathrm{H}_{117} \mathrm{~B}_{2} \mathrm{~N}_{12} \mathrm{O}_{4} \mathrm{Zn}_{2} \cdot \mathrm{C}_{6} \mathrm{H}_{14}$ & $\mathrm{C}_{110} \mathrm{H}_{122} \mathrm{~B}_{2} \mathrm{~N}_{12} \mathrm{O}_{4} \mathrm{Zn}_{2} \cdot\left(\mathrm{C}_{6} \mathrm{H}_{14}\right)_{1.5}$ & $\mathrm{C}_{105} \mathrm{H}_{118} \mathrm{~B}_{2} \mathrm{~N}_{12} \mathrm{O}_{5} \mathrm{Zn}_{2}$ \\
\hline Formula weight & 1837.74 & 1957.94 & 1780.58 \\
\hline Temperature (K) & $123(2)$ & $108(2)$ & 110 \\
\hline Wavelength & $0.71073 \mathrm{~A}$ & 0.71073 & 0.71070 \\
\hline Crystal system, space group & Monoclinic, $P 2(1) / c$ & Triclinic, $P 1$ & Triclinic, $P 1$ \\
\hline \multirow[t]{6}{*}{ Unit cell $(\AA)$} & $a=11.1192(12) \AA$ & $a=11.6009(10) \AA$ & $a=12.3136(5) \AA$ \\
\hline & $a=90^{\circ}$ & $a=76.390^{\circ}(5)$ & $\mathrm{a}=98.021^{\circ}(3)$ \\
\hline & $b=21.822(2) \AA$ & $b=18.5966(16) \AA$ & $b=13.0048(6) \AA$ \\
\hline & $\beta=94.885^{\circ}(7)$ & $\beta=85.934^{\circ}(6)$ & $\beta=95.060^{\circ}(3)$ \\
\hline & $c=44.040(5) \AA$ & $c=29.423(3) \AA$ & $c=33.3571(15) \AA$ \\
\hline & $\gamma=90^{\circ}$ & $\gamma=89.359^{\circ}(6)$ & $\gamma=97.950^{\circ}(2)$ \\
\hline Volume $\left(A^{3}\right)$ & $10647(2)$ & $6154(9)$ & $5207(4)$ \\
\hline$Z$, Calc density $\left(\mathrm{Mg} / \mathrm{m}^{3}\right)$ & $4,1.146$ & $2,1.057$ & $2,1.137$ \\
\hline$F(000)$ & 3908 & 2086 & 1888 \\
\hline Crystal size (mm) & $0.34 \times 0.24 \times 0.12$ & $0.34 \times 0.18 \times 0.16$ & $0.28 \times 0.20 \times 0.06$ \\
\hline$\theta$ range for data & $1.84^{\circ}$ to $22.10^{\circ}$ & $1.85^{\circ}$ to $22.16^{\circ}$ & $4.34^{\circ}$ to $44.12^{\circ}$ \\
\hline Reflections collected/unique & $127468 / 13121$ & $80150 / 15210$ & $18080 / 18080$ \\
\hline Completeness to $\theta$ & $99.3 \%$ & $98.5 \%$ & $99.3 \%$ \\
\hline Absorption Correction & Semi-emp from equiv & Semi-emp from equiv & Semi-empirical from equivalents \\
\hline Refinement method & Full-matrix least-squares on $F^{2}$ & Full-matrix least-squares on $F^{2}$ & Full-matrix least-squares on $F^{2}$ \\
\hline Data/restraints/param & $13121 / 0 / 1121$ & $15210 / 45 / 1196$ & $18080 / 0 / 1160$ \\
\hline Final $R$ indices $[I>2 \sigma(I)]$ & $\begin{array}{l}R 1=0.0858 \\
W R 2=0.1777\end{array}$ & $\begin{array}{l}R 1=0.0605 \\
w R 2=0.1545\end{array}$ & $\begin{array}{l}R 1=0.0614 \\
W R 2=0.1612\end{array}$ \\
\hline GOF & 1.092 & 0.993 & 1.064 \\
\hline$R$ indices (all data) & $R 1=0.1057, w R 2=0.1865$ & $R 1=0.1021, w R 2=0.1704$ & $R 1=0.0976, w R 2=0.1726$ \\
\hline $\begin{array}{l}\text { Largest difference peak } \\
\text { and hole }\end{array}$ & 0.970 and -0.700 e..$A^{-3}$ & 0.491 and -0.365 e. $A^{-3}$ & 0.656 and -0.556 e. $A^{-3}$ \\
\hline
\end{tabular}

crystallography within this series of complexes may be informative of substituent effects.

\section{UV-Vis spectroscopy}

To ascertain whether the change in the $n \rightarrow \pi^{*}$ transition correlates with $\sigma_{x}$ for $\operatorname{bis}\left(Z n^{\prime \prime}(S Q) T p^{\text {Cum,Me}}\right)$ complexes $\mathbf{5 a - 5}$, we calculated $\Delta v_{n} \rightarrow \Pi_{*}$ for the complexes where $\Delta v_{n} \rightarrow \Pi_{*}=v_{n} \rightarrow \Pi_{*}$ (5x) $-v_{n} \rightarrow \pi *(5 a)$ (Table 1$)$.

Correlations with a number of different $\sigma_{x}$ parameters with $\Delta v_{n} \rightarrow \pi_{*}$ values for the bis(Zn"(SQ)Tp ${ }^{\text {Cum,Me }}$ ) complexes were explored. Neither $\sigma ., \sigma-, \sigma_{\text {parar }} \sigma_{\text {meta }}$ nor the dual parameter $\sigma_{\mu}+\sigma_{x}$ system provided satisfactory correlations. This is not surprising because the gap between the two lowest states is a 
function of interactions amongst at least four different states the triplet state and the three lowest singlet states. Hence, substituents can alter the energies of the four lowest energy states and the singlet states can mix through both configuration interaction and second-order Jahn-Teller effects. ${ }^{[14]}$

\section{Electron paramagnetic resonance spectroscopy}

Exchange coupled biradical complexes have unique magnetic characteristics that manifest as anomalous absorbances in EPR spectrum. ${ }^{[15]}$ Most notable among these for the bis( $\mathrm{Zn}^{\text {II }}(\mathrm{SQ})$ $\mathrm{Tp}^{\mathrm{Cum}, \mathrm{Me}}$ ) complexes ( $S=1$ systems) is a triplet spin degeneracy arising from the dipole-dipole interaction of the unpaired electrons. In the absence of an applied magnetic field, all three sublevels of the triplet state are isoenergetic. However, infinitesimally small magnetic fields generated by the unpaired electrons lift the degeneracy in the triplet manifold. These intrinsic magnetic fields are operative regardless of the presence or absence of an applied field. Because no external field is necessary, the degeneracy lifting has been termed ZFS and is described using the parameters $D$ and $E$ (Fig. 5).

The ZFS parameter $D$ is an $\mathrm{e}^{-} / \mathrm{e}^{-}$repulsion energy and describes the deviation from cubic symmetry. $E$ is commonly referred to as the rhombic parameter and is a measure of the molecule's deviation from axial symmetry. ${ }^{[16]}$ Both $D$ and $E$ are anisotropic; therefore, the molecule must be restricted from freely tumbling to observe absorbances corresponding to the

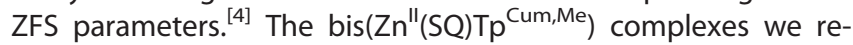
port give $D / h c$ and $E / h c$ values (Table 1) consistent with our previously published results. ${ }^{[1,12]}$ Values for the ZFS parameter $D$ obtained for compounds $\mathbf{5 a - c}$ do not vary significantly in magnitude within this series $\left(D / h c_{\text {avg }}=0.00389 \pm 0.00017\right)$. Thus, we can discern no substituent effect from this data.

\section{Ab initio computations}

Figure 6 depicts the spin density distributions for bis(semiquinone) [bis(SQ)] precursors to complexes $\mathbf{5 a - f}$ obtained by $a b$ initio methods. ${ }^{[17-21]}$ In Table 2, we record the gas-phase $a b$ initio spin densities at selected carbon centers and substituents for our bis(SQ) series. As the data show, calculated values for $\rho_{[C 7, C 8]}$ and $\Sigma \rho_{[\mathrm{C} 1-\mathrm{C6}]}$ re larger than the spin densities for the bis(SQ) precursor to 5a, implying a substituent effect. Furthermore, a significant proportion of the available spin density is retained in the meta-phenylene linker group. On average, over $20 \%$ of the spin density resides in this ring. Conversely, and somewhat unexpectedly, we see that substituents attached to the meta-phenylene ring retain spin densities ranging from $<0.2-2.5 \%$, a minimal proportion of that available.

We then investigated whether a correlation was present between spin densities at the bis(SQ) radical sites $C_{7}$ and $C_{8}, C_{1-6}$ and various Hammett $\sigma$-parameters, but no linear relationship was found.

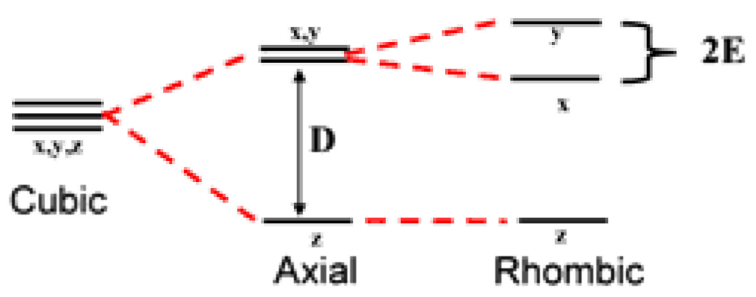

Figure 5. Splitting of formerly threefold degenerate triplet states
Reasons for the lack of a correlation include both structural and electronic considerations. In the crystal structures of the complexes, $C_{2}$ symmetry is evident, whereas gas-phase $a b$ initio results show bis(SQ) radical anion structures with the lower $C_{S}$ symmetry. Additionally, the computations do not account for interactions that the $\mathrm{Zn}^{\mathrm{II}} \mathrm{Tp}$ Cum,Me ligands may have on conformational changes or the electronic environment.

\section{Magnetometry}

A general trend consistent with earlier findings ${ }^{[1]}$ is evident in the data presented in Table 2. The magnitude of the exchange parameter $\boldsymbol{J}$ is related to the magnitude of the exchange integral. The data, taken as a whole, clearly show a twofold change in $\boldsymbol{J}$, as a function of substituent. We then used the method of Lahti ${ }^{[22]}$ to determine whether a relationship exists between the spin density, $\rho$, and $\boldsymbol{J}$ for our series of radicals by Eqn 2

$$
J_{\text {calc }}=J_{0} \rho^{\mathrm{A}} \rho^{\mathrm{B}}
$$

Estimation of the exchange parameter, $\boldsymbol{J}$, for the bis(semiquinone)s gives us a basis for comparison of our experimentally determined $\boldsymbol{J}$ values.

To determine $\boldsymbol{J}_{\text {calc }}$ for this series, the $a b$ initio-computed spin densities for the bis(SQ) precursors to $\mathbf{5 a - f}$ at $C_{7}$ and $C_{8}$ were substituted for $\rho^{A}$ and $\rho^{B}$, respectively. Calculated values for $\boldsymbol{J}$ using Eqn 2 range from approximately $20 \mathrm{~cm}^{-1}-34 \mathrm{~cm}^{-1}$. As Table 2 shows, $\boldsymbol{J}_{\exp }$ was within this range for complexes $\mathbf{5} \mathbf{d}$ and $\mathbf{5 e}$. The remaining complexes' $\boldsymbol{J}_{\text {exp }}$ values were larger than $\boldsymbol{J}_{\text {calc }}$ by factors ranging from 1.06-1.74. These variations are addressed below.

To determine empirically the nature of a substituent effect in the bis( $\left(\mathrm{n}^{\text {"I }}(\mathrm{SQ}) \mathrm{Tp}^{\mathrm{Cum}, \mathrm{Me}}\right)$ complexes proposed earlier, we investigated the relationship between the exchange parameter, $\boldsymbol{J}$, and various $\sigma$ constants. Correlations between $\boldsymbol{J}, \Delta, \log \left(\boldsymbol{J} / \mathbf{J}_{\mathbf{0}}\right)$, and a variety of Hammet $\sigma$ values including $\sigma_{l,} \sigma_{m}, \sigma_{.}, \sigma_{p}, \sigma^{+}$, and $\sigma_{\Delta v}$ were examined without success. Given this fact, it is difficult to deduce any empirical relationship between $\boldsymbol{J}$ and established $\sigma$ systems.

Earlier work has shown that $\boldsymbol{J}$ can be modulated by torsion angle. ${ }^{[12]}$ To determine whether this was the case for bis( $\mathrm{Zn}$ "(SQ) $\mathrm{Tp}^{\mathrm{Cum}, \mathrm{Me}}$ ) complexes, we investigated the possible correlation between $\boldsymbol{J}$ and the $m$-phenylene torsion angles $\varphi_{1}$ and $\varphi_{2}$ determined by X-ray crystallography (see Fig. 7 and Table 2). Correlations between $\left(\cos \varphi_{1}\right)^{2}\left(\cos \varphi_{2}\right)^{2}$ and $\boldsymbol{J}$ were examined, but were found to be unsatisfactory $(R<0.8)$. With the exception of $\mathbf{5} \mathbf{b}$, $\varphi_{\text {avg }}=32^{\circ} \pm 2$, a small variation within the series. Given these facts, it is difficult to assess any definitive modulation of $\boldsymbol{J}$ by complex conformation in this series.

Even with the apparent lack of any simple empirical relationship between $J$ and $\Delta v, a_{H}, \rho$ (spin density), known $\sigma$ parameters or conformational variations, the results from our study are consistent with previous work. ${ }^{[1]}$

The magnetic studies and $a b$ initio computations show that neither electron withdrawing groups, electron donors nor conjugating groups facilitate delocalization of the semiquinone radical anions into the central coupling meta-phenylene ring to increase the ferromagnetic exchange. In addition, our $a b$ initio results have revealed new information regarding the nature of spin density distribution in this system.

The simple Hückel molecular orbital theory argument posited in an earlier work by our group ${ }^{[1,8]}$ that mesomeric substituents attenuate the exchange coupling by spin density dilution relative 


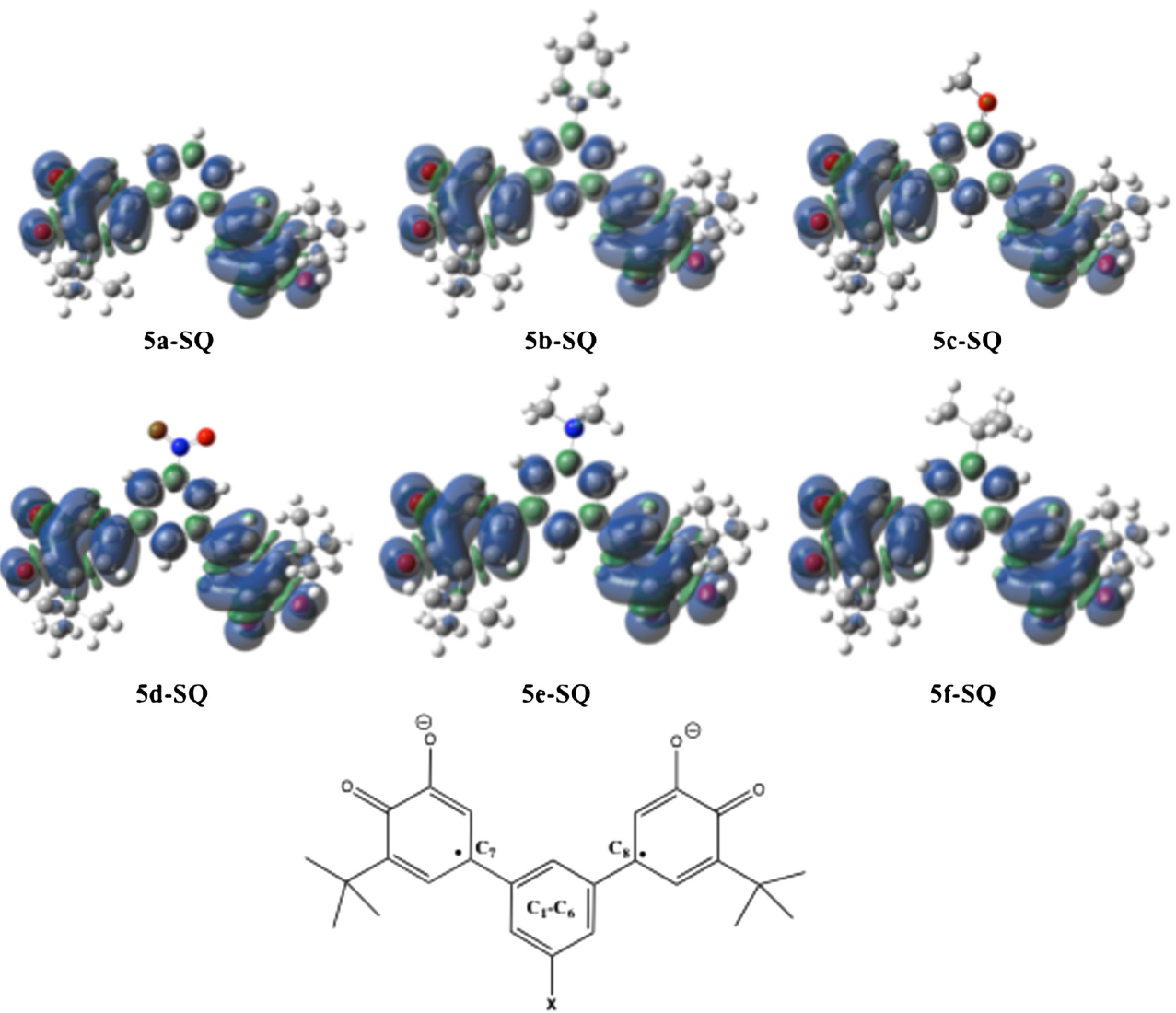

Figure 6. $\mathrm{Bis}(\mathrm{SQ})$ spin density distributions and atoms of interest (Blue $=\alpha$ spin density, green $=\beta$ spin density)

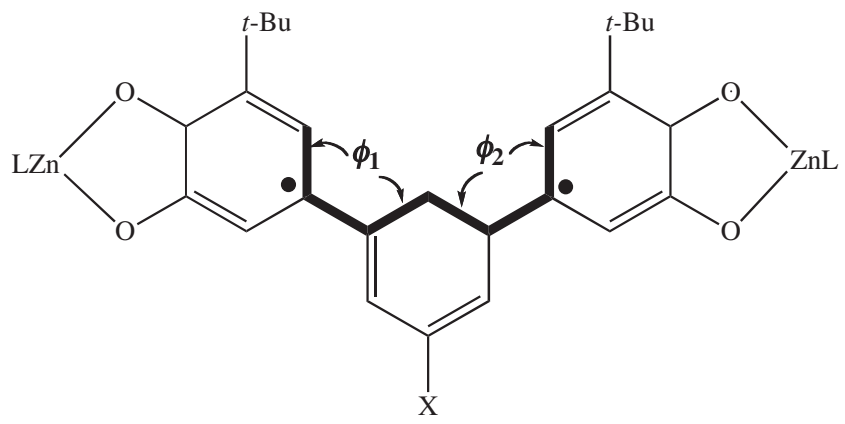

Figure 7. $m$-Phenylene torsion angles $\varnothing_{1}$ and $\varnothing_{2}$

to the unsubstituted meta-phenylene singly occupied molecular orbitals appears now to be incorrect, even though the correct trend was predicted. While this semi-empirical approach yielded serendipitous results, the more rigorous computations presented in this work show that the Hückel molecular orbit theory overestimates the delocalization of spin density into the 5-X-metaphenylene substituents.

\section{CONCLUSION}

Several new bis(Zn"(SQ)Tp ${ }^{\text {Cum,Me }}$ ) complexes to supplement the earlier series have been prepared and fully characterized. ${ }^{[1]}$ The results of the substituent study for these complexes indicate that there may be no straightforward empirical relationship between $\boldsymbol{J}$ and $\Delta v$, spin density, $\sigma$, or $m$-phenylene torsion. Our $a b$ initio computations show that substituents do not modulate spin density in the meta-phenylene linker ring by more than $10 \%$. Furthermore, calculations show negligible spin density on the phenylene substituents. This suggests that previous qualitative explanations of spin dilution by substituents that lead to exchange coupling attenuation were incidental.

Clearly, more effort is required to uncover the nature of the relationship between exchange coupling, spin density and substituents. Future work could include additional model systems that exhibit larger exchange couplings, so that comparisons might be made to the present work, and computations involving the bis $\left(\mathrm{Zn}^{\text {II }} \mathrm{SQ}\right) \mathrm{Tp}^{\mathrm{Cum}, \mathrm{Me}}$ ) complexes might hold even greater promise.

\section{EXPERIMENTAL}

\section{$A \boldsymbol{b}$ initio computations}

Initial geometry optimizations and frequency calculations were carried out for each molecule using the hybrid density functional Becke, three-parameter, Lee-Yang-Parr (B3LYP) ${ }^{[17,18]}$ and the 6$31 \mathrm{G}(\mathrm{d})$ basis set. After these initial optimizations, the structures were further refined using the EPR-II basis set of Barone et al. ${ }^{[19]}$ The EPR-II basis sets were optimized for the computation of hyperfine coupling constants with density functional theory. It was at these B3LYP/EPR-II geometries that the spin densities were computed at the same level of theory. Z-matrices of the optimized B3LYP/EPR-II structures and the corresponding harmonic frequencies can be found in the Supplementary Information. All calculations were performed with the GAUSSIAN 03 suite of $a b$ initio programs. ${ }^{[20]}$ All figures were generated with GAUsSVIEW 3.0. ${ }^{[21]}$ 


\section{Instrumentation}

Nuclear magnetic resonance (NMR) data were collected on a Varian VXR-300 NMR (Agilent Technologies, Inc. 5301 Stevens Creek BLVD., Santa Clara, CA, USA 95051). All ${ }^{1} \mathrm{H}$ and ${ }^{13} \mathrm{C}-\mathrm{NMR}$ chemical shifts reported are referenced to deuteriochloroform at the appropriate chemical shift unless specifically noted. Infrared data were collected using a Perkin-Elmer 1600 FT-IR spectrometer (Atlantic Microlab, Inc. 6180 Atlantic Blvd., Suitem, Noncross, GA 30071, USA). High resolution mass spectrometric analytical determinations were performed by the North Carolina State University Mass Spectral Facility. Elemental analyses were performed by Atlantic Microlab, Inc. Melting points were obtained on a Meltemp (Thermo Fisher Scientific, Inc. 81 Wyman Street, Waltham, MA 02454, USA) melting point apparatus and are uncorrected. X-band EPR spectra were recorded on an IBM-Bruker E200SRC spectrometer. Frozen solution EPR spectroscopy was performed on $1 \mathrm{mM}$ solutions of the $\operatorname{bis}\left(\mathrm{Zn}^{\text {II }}(\mathrm{SQ}) \mathrm{Tp}^{\mathrm{Cum}, \mathrm{Me}}\right)$ complexes in methyl tetrahydrofuran (THF). Magnetometry data was collected using a Quantum Design MPMSXL SQUID magnetometer (Quantum Design, Inc., 6325 Lusk Blvd., San Diego, CA 92121, USA).

\section{Chemicals}

1,3-Dibromobenzene was purchased from Aldrich Chemical Company (Sigma-Aldrich Corp. 3050 Spruce Street, St. Louis, MO 63103) and were used without further purification. Compounds $\mathbf{1} \mathbf{a}^{[23]} \mathbf{1} \mathbf{b}_{,}{ }^{[23]} 3,5$-dibromobiphenyl, ${ }^{[24]}$ and 3,5-dibromoanisole ${ }^{[25]}$ were prepared according to literature methods. All reactions, solvent distillations, and EPR sample preparation were conducted under nitrogen or argon atmosphere. Solvents were purchased from Fisher Scientific or Aldrich, and unless otherwise noted, were freshly distilled immediately prior to use. THF was distilled from sodium benzophenone-ketyl before use, while $\mathrm{CH}_{2} \mathrm{Cl}_{2}$ and $\mathrm{CH}_{3} \mathrm{OH}$ were distilled from $\mathrm{CaH}_{2}$.

\section{General procedure for the synthesis of bis(metaphenylene)s (3)}

To a Kjeldahl flask was added the 1,3-dibromoarene (1 eq), 1a or $\mathbf{1 b}(2 \mathrm{eq}), \mathrm{Pd}\left(\mathrm{PPh}_{3}\right)_{4}(5-10 \mathrm{~mol} \%)$, dry THF (25-50 mL), EtOH $(5-15 \mathrm{~mL}), 2 \mathrm{M} \mathrm{Na}_{2} \mathrm{CO}_{3}(7-22 \mathrm{~mL})$, and the reaction flask was purged three times with $\mathrm{N}_{2}$. To this was added a reflux condenser, the flask covered with aluminum foil and refluxed for 18-36h. The crude product was taken up in $\mathrm{CH}_{2} \mathrm{Cl}_{2}$ and filtered through Celite (Celite Corp. 2500 San Miguelito Road, Lompoc, CA 93436). This solution was washed with saturated $\mathrm{NaCl}$, dried over $\mathrm{Na}_{2} \mathrm{SO}_{4}$, and concentrated under reduced pressure. The resulting crude product was recrystallized or subjected to chromatography $\left(\mathrm{Et}_{2} \mathrm{O} / \mathrm{CH}_{2} \mathrm{Cl}_{2} /\right.$ P.E gradient elution), affording the bis (metaphenylene).

\section{General procedures for the preparation of catechols (4)}

\section{Method A}

A 100-mL round bottom flask equipped with a stir bar was charged with the t-butyl-bis(methoxymethyl)biphenyl ( 1-3 $\mathrm{mmol})$, concentrated $\mathrm{HCl}(2 \mathrm{~mL})$, and methanol $(50-70 \mathrm{~mL})$. A reflux condenser was attached, and the mixture refluxed for 18 $h$ with stirring under $\mathrm{N}_{2}$, protected from light. The solvent was removed by evaporation under reduced pressure. The residue was taken up in $\mathrm{CH}_{2} \mathrm{Cl}_{2}$, extracted with saturated brine, and the bottom organic layer dried over $\mathrm{Na}_{2} \mathrm{SO}_{4}$. The solvent was evaporated to dryness to afford the catechol.
Method B

A 125-mL Kjeldahl flask equipped with a $\mathrm{N}_{2}$ adapter and stir bar was charged with the $t$-butyl-dimethoxybiphenyl (1 equivalent, $\sim 0.5-1.5 \mathrm{mmol})$, distilled $\mathrm{CH}_{2} \mathrm{Cl}_{2}(\sim 10 \mathrm{~mL})$, purged three times with $\mathrm{N}_{2}$, and cooled to $-78{ }^{\circ} \mathrm{C}$. Then, a $1.0 \mathrm{M}$ solution of $\mathrm{BBr}_{3}$ (3 equivalents, $\sim 1.5-4.5 \mathrm{mmol}$ ), was added dropwise slowly to the reaction mixture, which was then allowed to warm to room temperature and stir for $20 \mathrm{~h}$ under $\mathrm{N}_{2}$, protected from light. The reaction mixture was quenched with ice, washed with saturated brine and extracted with two $10 \mathrm{~mL}$ portions of $\mathrm{CH}_{2} \mathrm{Cl}_{2}$. The organic layers were combined, dried over $\mathrm{Na}_{2} \mathrm{SO}_{4}$, and the solvent evaporated to dryness to afford the catechol.

\section{General procedure for the synthesis of $\mathrm{Bis}\left(\mathrm{Zn}^{\text {"(SQ)Tp }}{ }^{\mathrm{Cum}, \mathrm{Me}}\right)$ complexes (5)}

A 100-mL round bottom flask was charged with 1 equivalent $\mathrm{KTp}{ }^{\text {Cum, Me }}(\sim 0.2 \mathrm{mmol}), 30 \mathrm{~mL}$ distilled $\mathrm{CH}_{2} \mathrm{Cl}_{2}, 1$ equivalent $\mathrm{Zn}$ $\left(\mathrm{ClO}_{4}\right)_{2} \cdot 6 \mathrm{H}_{2} \mathrm{O}(\sim 0.2 \mathrm{mmol})$, and $4.5 \mathrm{~mL} \mathrm{~N}_{2}$ saturated methanol. The mixture was stirred until all reagents dissolved (15-30 min). Then, 0.5 equivalents of the bis(catechol) $(\sim 0.1 \mathrm{mmol})$ was added to the flask and the reaction mixture stirred under $\mathrm{N}_{2}$ for an additional $1 \mathrm{~h}$. Finally, triethylamine $(0.695 \mathrm{~mL}$ of a $4 \%$ solution v:v with $\mathrm{CH}_{2} \mathrm{Cl}_{2}$ ) was delivered via syringe into the reaction flask and the mixture stirred for an additional $1.5 \mathrm{~h}$. The reaction flask was opened to air and stirred overnight. The greenish-brown solution was concentrated under reduced pressure. The resulting solid was washed with diethyl ether, filtered, and the solvent removed under reduced pressure. The resulting microcrystalline product was recrystallized, affording the complex.

\section{SUPPORTING INFORMATION}

Experimental methods, $1 \mathrm{H}$ and $13 \mathrm{C}$ spectral data, analytical data, $\mathrm{ab}$ initio parameters, and x-ray crystallographic parameters for reported compounds. This material is available free of charge via the online version of the paper. The crystal structural data have been deposited in the Cambridge Crystallographic Data Center and were assigned the following numbers: complex 5a: CCDC 817203, complex 5b: CCDC 817205, complex 5c: CCDC 817204.

\section{Acknowledgements}

The authors would like to thank the NCSU X-Ray Crystallographic Facility for X-ray analysis and the NCSU Mass Spectroscopy Facility for high resolution MS analysis. The authors gratefully acknowledge Professor Joel M. Bowman (Emory University) for providing the computer resources used to carry out this work. DAS thanks the NSF (CHE-0345263) for support of this work.

\section{REFERENCES}

[1] D. A. Shultz, S. H Bodnar, H. Lee, J. W. Kampf, C. D. Incarvito, A. L. Rheingold, J. Am. Chem. Soc. 2002a, 124, 10054.

[2] D. A. Shultz, S. H Bodnar, K. E. Vostrikova, H-J. Koo, M.-H. Whangbo, M. Kirk, E. Depperman, J. W. Kampf, J. Chem. Soc. 2003a, 125, 1607.

[3] Bruker WINEPR Simfonia, 1.25, Shareware Version, Bruker Analytische Messtechnik GmbH, Rheinstetten, Germany, 1996.

[4] O. Kahn, Molecular Magnetism, VCH Publishers, Inc, New York, 1993.

[5] B. B. Bleaney, Proc. R. Soc. Lond. 1952, A214, 451.

[6] C. J. O'Connor, Prog. Inorg. Chem. 1982, 29, 203.

[7] A. D. Caneschi, C. P. Mussari, D. A. Shultz, L. Sorace, K. E. Vostrikova, Inorg. Chem. 2002, 41, 1086. 
[8] W. T. Borden, R. R. Squires, C. R. Kemnitz, J. Am. Chem. Soc. 1997 $119,6564$.

[9] W. T. Borden, H. Iwamura, J. A. Berson, Acc. Chem. Res. 1994, 27, 109.

[10] E. R. Davidson, W. T. Borden, J. Am. Chem. Soc. 1977, 99, 4587.

[11] W. T. Borden, E. R. Davidson, Acc. Chem. Res. 1981, 14, 69.

[12] D. A. Shultz, R. M. Fico, S. H. Bodnar, R. K. Kumar, K. E. Vostrikova, J. W. Kampf, P. D. Boyle, J. Am. Chem. Soc. 2003b, 125, 11761-11771

[13] C. K. Hancock, D. Clague, J. Am. Chem. Soc. 1964, 86, 4942.

[14] D. A. Shultz, R. M. Fico, S. H. Bodnar, R. K. Kumar, K. E. Vostrikova, J. W. Kampf, P. D. Boyle, J. Am. Chem. Soc. 2002b, 124, 10054

[15] J. E. Wertz, J. R. Bolton, Electron Spin Resonance, Chapman and Hall, 1986.

[16] H. K. Kurreck, B. Kirste, W. Lubitz, Electron Nuclear Double Resonance Spectroscopy of Radicals in Solution, VCH Publishers, Berlin, 1988.

[17] A. D. Becke, J. Chem. Phys. 1993, 98, 5648.

[18] P. J. Sephens, F. J. Devlin, C. F. Chabalowski, M. J. Frisch, J. Phys. Chem. 1994, 98, 11623.

[19] V. Barone, in: Recent Advances in Density Functional Methods, Part I, (Ed: D. P. Chong), World Scientific Publ. Co, Singapore, 1996.

[20] M. J. Frisch, G. W. Trucks, H. B. Schlegel, G. E. Scuseria, M. A. Robb, J. R. Cheeseman, J. A. Montgomery Jr., T. Vreven, K. N. Kudin, J. C. Burant, J. M. Millam, S. S. Iyengar, J. Tomasi, V. Barone, B. Mennucci,
M. Cossi, G. Scalmani, N. Rega, G. A. Petersson, H. Nakatsuji, M. Hada, M. Ehara, K. Toyota, R. Fukuda, J. Hasegawa, M. Ishida, T. Nakajima, Y. Honda, O. Kitao, H. Nakai, M. Klene, X. Li, J. E. Knox, H. P. Hratchian, J. B. Cross, V. Bakken, C. Adamo, J. Jaramillo, R. Gomperts, R. E. Stratmann, O. Yazyev, A. J. Austin, R. Cammi, C. Pomelli, J. W. Ochterski, P. Y. Ayala, K. Morokuma, G. A. Voth, P. Salvador, J. J. Dannenberg, V. G. Zakrzewski, S. Dapprich, A. D. Daniels, M. C. Strain, O. Farkas, D. K. Malick, A. D. Rabuck, K. Raghavachari, J. B. Foresman, J. V. Ortiz, Q. Cui, A. G. Baboul, S. Clifford, J. Cioslowski, B. B. Stefanov, G. Liu, A. Liashenko, P. Piskorz, I. Komaromi, R. L. Martin, D. J. Fox, T. Keith, M. A. Al-Laham, C. Y. Peng, A. Nanayakkarra, M. Challacombe, P. M. W. Gill, B. Johnson, W. Chen, M. W. Wong, C. Gonzalez, J. A. Pople, Gaussian 03, Revision C.01, Gaussian, Inc., Wallingford, CT, 2004.

[21] R. Dennington, II; T. Keith, J. Millam, K. Eppinnett, W. L. Hovell, R. Gilliland, GaussView, Version 3.0, Semichem, Inc, Shawnee Mission, KS, 2003.

[22] P. Lahti ed., Magnetic Properties of Organic Materials, Marcel Dekker, Inc, New York, 1999, p. 253.

[23] D. A. Shultz, A. K. Boal, D. J. Driscoll, G. T. Farmer, J. R. Kitchin, D. B. Miller, G. N. Tew, Mol. Cryst. Liq. Cryst. 1997, 305, 303.

[24] T. Kelly, G. Bridger, C. Zhao, J. Am. Chem. Soc. 1990, 112, 8024.

[25] L. Dorman, J. Org. Chem. 1966, 31, 3666. 